\title{
EDITORIAL
}

\section{Fetal maternal immunity and antileukemia activity in cord-blood transplant recipients}

Bone Marrow Transplantation (2013) 48, 321-322; doi:10.1038/ bmt.2012.157; published online 24 September 2012

Umbilical cord blood has become a viable and widely used alternative stem cell source for patients with hematologic diseases in need of a hematopoietic cell transplant. ${ }^{1-4}$ Several studies have reported comparable survival rates for adult cord-blood transplant (CBT) recipients when compared with unrelated donor peripheral blood or BMT. ${ }^{5,6}$ Moreover, Brunstein et al. ${ }^{7}$ recently reported a lower rate of relapse among double CBT recipients than patients who received either matched or mismatched unrelated donor transplants. The vast majority of CBT recipients receive highly HLAmismatched grafts, which may contribute to the lower incidence of relapse. Interestingly, a recent study suggests maternal cells in CB grafts that are sensitized to fetal inherited paternal antigens (IPAs) (Figure 1a) may also contribute to the lower incidence of relapse. $^{8}$

Fetal exposure to noninherited maternal HLA antigens (NIMA) (Figure 1b) is thought to produce tolerance to NIMA, and improved outcome was previously reported when NIMA of the CB donor was shared by a recipient. ${ }^{9}$ On the other hand, during pregnancy women develop B- and T-cell immunity against fetal histocompatibility antigens and minor histocompatibility antigens that are paternally-inherited. Although CB grafts consist primarily of fetal cells, some maternal cells are present. ${ }^{10,11}$ Indirect evidence reported by van Rood et al. ${ }^{8}$ strongly implicates maternal immune cells with anti-IPA activity in the GVL effect observed with CBT. Maternal cells are implicated because relapse was reduced specifically when the transplant recipient had one or more HLA antigens that were the same as an IPA of the CB donor. The study included patients with hematological malignancies $(n=1155)$ who were transplanted with a single $C B$ unit from the New York Cord Blood Bank between 1993 and 2006. The majority of patients $(n=1030)$ shared one or more HLA antigens with CB IPAs and had at least 1, 2 or 3 HLA-A, -B, -DRB1 mismatches. No differences, with respect to total nucleated cell dose and patient characteristics, were seen between no-shared IPA $(n=64)$ and shared-IPA transplants. Interestingly, the risk of relapse within the first 3 years was significantly lower among the patients with shared IPA than those that with no-shared IPA. The risk reduction was stronger in patients who received CBT that had one HLA mismatch. In this group of patients lower relapse risk was associated with a lower risk of treatment failure, although without significant improvement in overall survival.

Of note, van Rood et al. also showed that the risk of relapse was lower when the CB unit came from a later birth order versus a first birth, further supporting a role of maternal exposure to fetal paternally-inherited HLA-antigens. In addition, when HLA mismatch of the mother and the CBT recipient was considered in a multivariate model, the association between shared IPA and relapse risk reduction remained significant. The latter analysis confirmed that the GVL effect could not be attributed to maternal mismatch but was putatively due to maternal cells with anti-IPA activity. The price to pay for increased GVL activity was a slightly higher risk of acute grade III-IV GVHD among patients with shared IPAs. However, GVHD incidence was only significant in patients with two HLA mismatches, and NS in patients with only one HLA mismatch. a
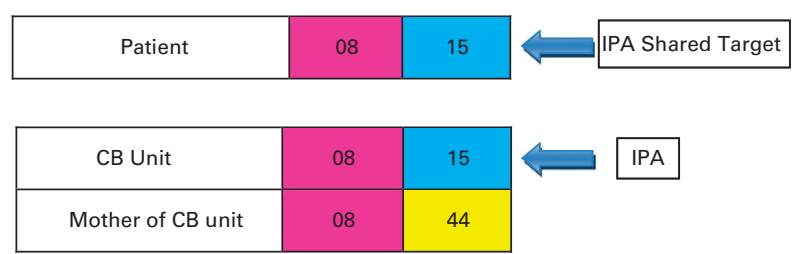

b

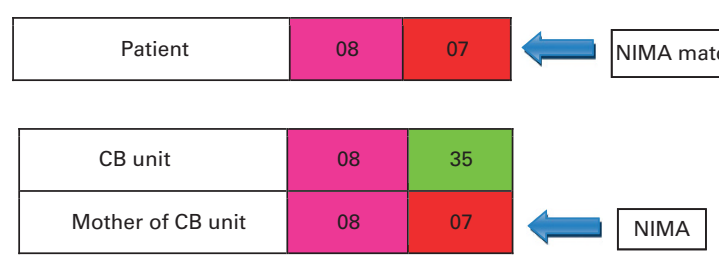

Figure 1. (a) HLA-B typing is used as an example to illustrate the IPA in cord blood transplantation. Antirelapse benefit is predicted in the example illustrated because the mother's T cells would be sensitized to the HLA-B Ag encoded by $B^{*} 15$ (IPA) and the CB recipient has $B^{*} 15$ (shared IPA target). Patient and donor $C B$ unit match for $B$ antigens encoded by $B^{*} 08$ and $B^{*} 15$. Based on the HLA typing of the $C B$ and its mother, $B^{*} 15$ is the IPA in the CB. In this case, the patient also has a $B^{*} 15$, so IPA-sensitized maternal cells have a target. (b) Decreased transplant related mortality was reported in an earlier paper ${ }^{9}$ for patients receiving one or two Ag-mismatched CBTs when the patient had the same HLA Ag as the CB's NIMA. Patient and donor $C B$ unit are mismatched at the $B$ locus: patient has $B^{*} 07$ while $C B$ has $B^{*} 35$. However, the patient's mismatched $B^{*} 07$ matches the CB's NIMA at the same locus.

This important work brings a new perspective to the way we think of the immunological potential of CBT. The antileukemia activity of CB grafts is likely due at least in part to maternal immune cells contained within the graft conferring a potent GVL effect. However, this indirect evidence needs now to be followed by specific work that identifies and characterizes the type of maternal cells in CB and the mechanism of action for the observed GVL effect. From a practical standpoint, the evaluation of IPA match in CB selection can be considered at the time a CB donor search is initiated. Although the majority of patients will share one or more $\mathrm{Ag}$ with CB IPAs, the benefit of knowing IPAs along with NIMA is likely to improve CB unit selection and consequently to significantly enhance the efficacy of CBT for patients with hematological malignancies.

\section{CONFLICT OF INTEREST}

The authors declare no conflict of interest.

F Milano ${ }^{1}$, J Lee Nelson ${ }^{1,2}$ and C Delaney ${ }^{1,3}$ ${ }^{1}$ Department of Pediatric Oncology, Clinical Research Division, Fred Hutchinson Cancer Research Center, Seattle, WA, USA; ${ }^{2}$ Department of Medicine University of Washington, Seattle, WA, USA and

${ }^{3}$ Department of Pediatrics, UW, Seattle, WA, USA E-mail: fmilano@fhcrc.org 


\section{REFERENCES}

1 Laughlin MJ, Barker J, Bambach B, Koc ON, Rizzieri DA, Wagner JE et al. Hematopoietic engraftment and survival in adult recipients of umbilical-cord blood from unrelated donors. N Engl J Med 2001; 344: 1815-1822.

2 Laughlin MJ, Eapen M, Rubinstein P, Wagner JE, Zhang MJ, Champlin RE et al. Outcomes after transplantation of cord blood or bone marrow from unrelated donors in adults with leukemia. N Engl J Med 2004; 351: 2265-2275.

3 Rocha V, Labopin M, Sanz G, Arcese W, Schwerdtfeger R, Bosi A et al. Transplants of umbilical-cord blood or bone marrow from unrelated donors in adults with acute leukemia. N Engl J Med 2004; 351: 2276-2285.

4 Kurtzberg J, Laughlin M, Graham ML, Smith C, Olson JF, Halperin EC et al. Placental blood as a source of hematopoietic stem cells for transplantation into unrelated recipients. N Engl J Med 1996; 335: 157-166.

5 Eapen M, Rubinstein P, Zhang MJ, Stevens C, Kurtzberg J, Scaradavou A et al. Comparison of outcomes after transplantation of unrelated donor umbilical cord blood and bone marrow in children with acute leukemia. Lancet 2007; 369: 1947-1954.

6 Gutman JA, Leisenring W, Appelbaum FR, Woolfrey AE, Delaney C. Low relapse without excessive transplant-related mortality following myeloablative cord blood transplantation for acute leukemia in complete remission: a matched cohort analysis. Biol Blood Marrow Transplant 2009; 15: 1122-1129.

7 Brunstein C, Gutman JA, Weisdorf DJ, Woolfrey AE, Defor TE, Gooley TA et al. Allogeneic hematopoietic cell transplantation for hematological malignancy: relative risk and benefits of double umbilical cord. Blood 2010; 116: 4693-4699.

8 van Rood J, Scaradavou A, Stevens C. Indirect evidence that maternal microchimerism in cord blood mediates a graft-versus-leukemia effect in cord blood transplantation. Proc Natl Acad Sci 2012; 109: 2509-2514.

9 van Rood J, Stevens C, Smits J, Carrier C, Carpenter C, Scaradavou A. Exposure of cord blood to noninherited maternal HLA antigens improves transplant outcome in hematological malignancies. Proc Natl Acad Sci 2009; 106: 19952-19957.

10 Lo YMD, Lo SF, Watson N, Noakes L, Sargent IL, Thilaganathan B et al. Two-way cell traffic between mother and fetus: biologic and clinical implications. Blood 1996; 88: 4390-4395.

11 Mold J, Michaelsson J, Burt T, Muench M, Beckerman K, Busch M et al. Maternal alloantigens promote the development of tolerogenic fetal regulatory $\mathrm{T}$ cells in utero. Science 2008; 322: 1562-1565. 